Article

\title{
Performance of a Thermoelectric Generator Partially Illuminated with Highly Concentrated Light
}

\author{
Abdelrahman Lashin ${ }^{1,2}$, Mohammad Al Turkestani ${ }^{1}$ and Mohamed Sabry ${ }^{1,3, *}$ \\ 1 Physics Department, College of Applied Science, Umm Al Qura University, Makkah 21955, Saudi Arabia; \\ aylashin@uqu.edu.sa (A.L.); mkturkestani@uqu.edu.sa (M.A.T.) \\ 2 Physics Department, Faculty of Science, Mansoura University, Mansoura 35516, Egypt \\ 3 Solar Physics Lab, National Research Institute of Astronomy and Geophysics, Cairo 11421, Egypt \\ * Correspondence: mmsalaheldin@uqu.edu.sa
}

Received: 31 May 2020; Accepted: 11 July 2020; Published: 14 July 2020

check for updates

\begin{abstract}
In order to maximize the output of concentrator Photovoltaic cells and maintain their efficiencies, the operating temperature of concentrator photovoltaic cells must be reduced. A way that could reduce such photovoltaic temperature is by thermally attaching them on top of a thermoelectric generator. A thermoelectric generator in such coupling will act as a low-cost passive-cooling subsystem, as well as a power generator for producing additional energy from the rejected photovoltaic heat. Increasing the area of the proposed photovoltaic cells relative to the thermoelectric generator's hot-side area will result in an increase in the thermoelectric generator's electrical output, but may also result in overheating the photovoltaic cells, hence reducing their performance. Optimization has to be performed for the photovoltaic covering percentage relative to the hot-side area in order to maximize the output of the whole coupled system. This work investigates the electrical and thermal performance of thermoelectric generators in the case of partial illumination of their hot side. Experiments have been carried out using three thermoelectric generator modules with different areas, and under two levels of concentrated illumination. The thermoelectric generator output voltage, current, and temperature have been measured, and the figure-of-merit and maximum power of the thermoelectric generator (TEG) has been calculated and demonstrated.
\end{abstract}

Keywords: concentrator photovoltaic; thermoelectric generator; PV/T hybrid system; CPV/TEG hybrid system

\section{Introduction}

It is a fact that flat-plate modules is very cheap, but its efficiency is around $23 \%$ for single junction panels, except in laboratory cells under examination. In contrast, concentrator photovoltaic cell (CPV) efficiencies are much higher; an example is a recently announced six-junction solar cell that already exceeded $47 \%$ under 143 sun, and around $45 \%$ under 1000 sun [1]. Thus, it is believed that concentrator photovoltaic cells (CPV) are superior to flat-plate ones. This will certainly reflect on the cost/watt of the CPV systems.

Employing such concentrated solar radiation will have two effects: (1) increasing solar radiation intensity will reduce the cost of the whole system, including the PV cell and the required spaces outdoors; (2) the necessity for reducing CPV cell temperature by a passive or active cooling subsystem in order to maintain a proper output. The former cooling subsystem is avdantageous, while the latter, although more efficient, requires added costs to the system (disadvantage).

Reducing cell temperature is a challenge in such CPV systems. Although the current increases slightly with temperature elevation, the voltage drops considerably [2], which results in reducing the fill factor, output power, and PV efficiency $[3,4]$. Such drawbacks necessitate implementing cooling 
techniques in the CPV system. Although active cooling systems reduce CPV cell temperature efficiently, they require a complicated operation and consume energy to operate. In comparison, passive cooling subsystems operate at no cost, but are not as efficient as active subsystems.

Thermoelectric generators (TEG), on the other hand, are semiconductor devices capable of generating electrical power when exposed to a temperature difference between its surfaces. They are reliable and withstand high thermal flux. Attempts to concentrate sunlight directly on TEG modules have been tested, and a system efficiency of up to $14 \%$ has been predicted [5].

Several studies have been carried out on the feasibility of coupling TEGs to PV. A beam splitter was designed to separate infrared radiation from the visible spectrum, and it directs this infrared band to a TEG module, while directing the rest of the spectrum to be incident on PV cells [6]. Another study has dealt with thermoelectric generators combined with the effects of heat sensitive materials associated with photovoltaic cells in phase change for generating electricity over the whole day [7]. In other articles [8,9], a solar concentrating thermoelectric generator using a micro-channel heat pipe array was designed in addition to a simulation model. The results showed a higher performance when using such a micro-channel heat pipe array system, including higher heat-transfer performance, low thermal contact resistance, higher output performance and economic value. In another research [10], a CPV-TE hybrid system 3D simulation with moth-eye nanostructures and a linear Fresnel reflective solar concentrator was established. In this work, a novel way of using different dimensional nanostructures to improve the uniformity of the absorbed irradiance distribution was proposed.

A proposed CPV/TEG coupled system [11] is composed of a large area Fresnel lens for concentrating solar radiation on a solar cell that is passively cooled by thermally attaching it to the top side of a TEG unit such that it covers only part of the top of the TEG surface. The TEG's bottom (cold) side is again thermally attached to a heat sink, which maintains a temperature difference between the top (hot) and the bottom (cold) sides of the TEG. In a recent work [11] a CPV/TEG hybrid system has been tested against two different CPV cells, namely silicon LGBC and multijunction $\mathrm{Ga}_{0.35} \mathrm{In}_{0.65} \mathrm{P} / \mathrm{Ga}_{0.83} \mathrm{In}_{0.17}$ solar cells. The results revealed that using the multijunction solar cell showed a withstanding electrical output under high levels of concentrated light, as well as additional output power generated by the TEG.

The concentrated solar radiation of power $Q_{\text {rad }}$ received by a CPV cell could be expressed as:

$$
Q_{\text {rad }}=I_{\text {rad }} \cdot A_{\text {lens }} \eta_{\text {lens }}
$$

where $I_{\text {rad }}$ is solar radiation intensity incident on the whole surface area of a concentrating lens $A_{\text {lens }}$ and optical efficiency $\eta_{\text {lens }}[12,13]$. This incident solar radiation power is partly converted into electricity $Q_{\text {elec }}$, while the remaining part of the solar power is dissipated as heat $Q_{\text {th }}$ inside the CPV cell, and is determined as [13]:

$$
Q_{\text {th }}=Q_{\text {rad }}-Q_{\text {elec }}=I_{\text {rad }} \cdot A_{\text {lens }} \cdot \eta_{\text {lens }}\left(1-\eta_{\text {sc }}\right),
$$

where $\eta_{s c}$ is the CPV cell electrical efficiency.

If such a CPV cell is brought in thermal contact with the hot side of a TEG, this dissipated heat will be then transferred to the TEG, and additional thermoelectricity is generated. The idea is to use the TEG as a passive cooling device for the CPV, under the condition that the CPV temperature is kept as low as possible under high-incident radiation. Otherwise, the total CPV/TEG will be lower than expected.

To increase the CPV/TEG system electrical output, one can increase the intensity of concentrated light incident on the CPV cell, and also increase the area of the CPV covering the TEG hot side.

It is obvious that increasing the area of the CPV cells thermally attached on top of the TEG's hot side (i.e., increasing the area covered by CPV cells relative to the total TEG hot-side area) will result in an increase in the energy input to the CPV/TEG system, leading to generating more thermoelectric power. On the other hand, it is known that PV efficiencies are much higher than those of the TEGs. A factor that will definitely reduce the whole CPV/TEG system is that the elevation in PV temperature 
is not accompanied by the ability of the TEG to dissipate such high temperature by converting it into electricity. This will result in increasing the temperature of the PV cells to levels that greatly impair its efficiency. Assuming the CPV area is fixed, attaching it to a bigger TEG will definitely dissipate the CPV temperature more efficiently compared to a smaller TEG, but will generate lower thermoelectric power, because its covering percentage is lower than that of the smaller TEG.

For maximizing the total electrical output of the proposed CPV/TEG coupled system, different parameters have to be optimized:

i. The area and efficiency temperature coefficient of the CPV attached on top of the TEG.

ii. The area of the TEG to be used as heat dissipation and thermoelectric generation.

iii. The operating light intensity.

iv. The overall system temperature.

This work focuses on investigating the performance of a TEG under partial illumination over its top surface with different covering percentages, as well as different light intensities in the case of partial illumination.

\section{Characterization of TEG Devices}

Thermal energy harvesting using a TEG is an economically efficient way to generate green energy. A TEG converts heat directly into electrical energy according to the Seebeck effect. However, energy conversion efficiency of such devices is lower than those of convectional heat engines. The efficiency of TEGs and the coefficient of performance (COP) are functions of not only the figure-of-merit (ZT), but also of the temperature difference between their hot and cold sides [14].

The efficiency of a TEG is given by [15]

$$
\eta=\frac{P_{\text {out }}}{Q_{\text {in }}}
$$

where $P_{\text {out }}$ is the extracted electric output power and $Q_{\text {in }}$ is the net heat flowing into the TEG.

A measure of the overall performance of the TEG is its figure-of-merit, ZT, given by Equation (4). It relates the TEG's performance to its material physical properties [16].

$$
\mathrm{ZT}=\frac{\alpha^{2} T}{\rho k}
$$

where $\alpha$ is the Seebeck coefficient, $\rho$ is the electrical resistivity, $k$ is the thermal conductivity, and $T$ is the operating temperature.

Theoretical conversion efficiency of such a TEG may be calculated from [15]

$$
\eta=\frac{T_{H}-T_{C}}{T_{H}} \frac{\sqrt{1+Z T}-1}{\sqrt{1+Z T}+\frac{T_{C}}{T_{H}}}
$$

where $T_{C}$ and $T_{H}$ are the cold- and hot-side temperature, respectively.

Equation (3) cannot be used directly for calculating TEG efficiency due to the difficulties in measuring the input heat flowing to the TEG $Q_{i n}$ accurately. Experimental determination of such net input heat requires determining the amount of heat transferred by conduction, which requires knowing thermal properties and the thickness of the thermal grease used as the interface layer. Furthermore, thermal losses in the form of convective and radiative heat transfer to the surrounding environment are quite difficult tasks to calculate, especially at operating temperatures exceeding $200{ }^{\circ} \mathrm{C}$.

Different methods have been proposed for measuring TEG efficiency. The Harman method [17] is used to characterize the TEG's performance over a small temperature difference between its hot and cold sides under an adiabatic condition (a condition that requires no heat loss or transfer from the system to the surroundings). This method is applicable for 10 to $20^{\circ} \mathrm{C}$ temperature difference 
between the TEG's hot and cold sides. Additionally, this method assumes constant values of $\alpha, \rho$ and $k$ with temperature, an assumption that cannot be applied in real TEG operating conditions. Others developed a method to characterize a single TE leg under high-temperature differences [15]. A theoretical determination of figure-of-merit was proposed by measuring the temperature differences in the open- and short-circuit states of the TEG at steady-state conditions, rather than the adiabatic conditions required in the Harman method [18]. Another method has been proposed for determining thermoelectric properties from the current-voltage curves under constant temperature differences and constant thermal input flux [19]. The results obtained in the study confirm the validity of this method. In addition, using this method allows measuring the ZT under a large temperature difference between the TEG's hot and cold sides. A similar method has been proposed [20] for estimating the maximum power, efficiency and the ZT of the TEG at two different steady state thermal conditions. This method has been adopted in this work, and will be discussed in more detail later on.

\section{Measurement and Calculation Method}

In this work, three different square-shaped TEGs manufactured by European Thermodynamics have been used, whose dimensions and number of junctions are listed in Table 1.

Table 1. Side length and number of junctions of the thermoelectric generator (TEG) samples investigated.

\begin{tabular}{ccc}
\hline TEG Sample & Side Length $(\mathbf{c m})$ & Number of Junctions \\
\hline A & 3 & 31 \\
B & 4 & 127 \\
C & 6.2 & 49 \\
\hline
\end{tabular}

Concentrated light was produced by a Xenon-Lamp solar simulator with intensities of $12 \times$ and $105 \times$. The output voltage and current of each of the TEGs have been measured under these two illumination levels.

The area of illumination was controlled by using a series of galvanized steel shadow masks with holes of various diameters. These masks were used to cover the top side of each of the TEGs under examination without being physically contacted to the top of its surface. This ensures that only a spot of concentrated light is incident on a part of the TEG top surface area, while the remaining area is covered. The bottom side of the TEG is thermally attached to a heat sink that is immersed in a water bath containing water flowing at constant temperature of $23^{\circ} \mathrm{C}$. Figure 1 shows a photo of the setup used in the experiment, with an illustration of the partial illumination technique used.

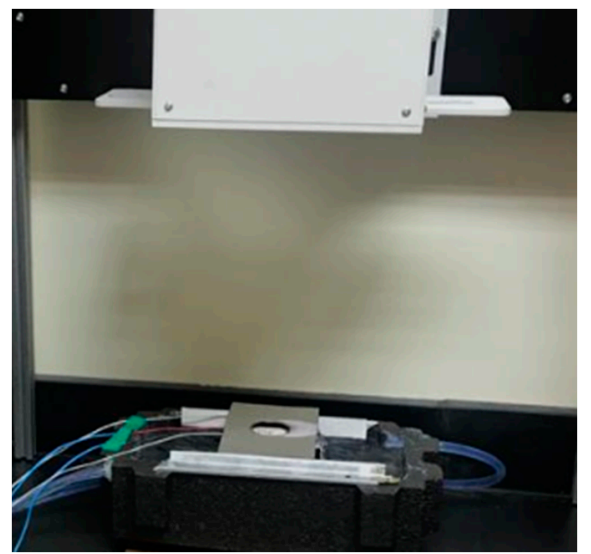

(a)

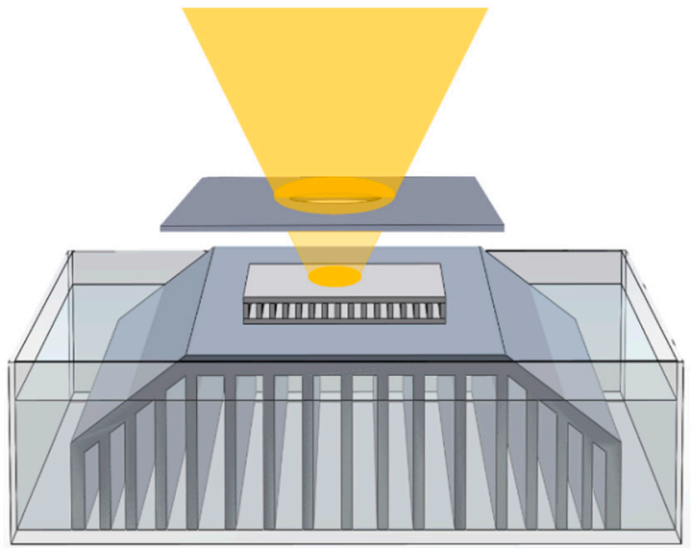

(b)

Figure 1. Photo (a) and sketch (b) of the setup used. 
The method adopted in this work for estimating the TEG properties is based on measuring the open-circuit voltage, the short-circuit current as well as the temperature difference between the TEG sides at two different steady state thermal conditions. Those two conditions are (1) electrically open-circuited terminals and (2) electrically short-circuited terminals. The heat flow into the TEG module is tuned by means of changing the intensity of the incident concentrated light $\left(Q_{i n}\right)$ so that the hot-side and cold-side temperatures are adjusted to be the same in both thermal conditions. The process of acquiring TEG output is composed of recording four subsequent pairs of data points as in the following steps [20]:

$i$. The current, $I_{S}$, is measured (the switch is in position 1 in Figure 2) after reaching the desired cold-side temperature, $T_{c}$, and the hot-side temperature, $T_{h}$, by means of adjusting $Q_{i n}$ and achieving a steady-state condition. This corresponds to the zero open-circuit voltage (the TEG is in the short-circuit condition).

ii. The voltage, $V_{s \rightarrow o}$ (short- to open-circuit voltage), is measured promptly after opening the circuit (the switch moves from position 1 to position 2 in Figure 2), i.e., in the transient condition between the short- and the open-circuit condition. After this prompt the recording of $V_{s \rightarrow 0}$, as the TEG current falls to zero (because of the open-circuit condition) a fast increase in the voltage will be observed. This is because of the change in the temperature difference between the TEG sides.

iii. In the steady-state open-circuit condition, $Q_{i n}$ is re-adjusted (by tuning the light intensity of the solar simulator) such that $T_{c}$ and $T_{h}$ are set back to the same values as those in the first step. After establishing thermal equilibrium, the voltage, $V_{o}$, is measured (the switch is in position 2 in Figure 2). This corresponds to zero current (i.e., $I_{0}=0$ because of the open-circuit condition).

iv. Finally, the circuit is closed (the switch is set back to position 1 in Figure 2), and the current, $I_{0 \rightarrow s}$ (open-to-short-circuit current), is measured immediately before any changes occur to the TEG module temperatures. In this paper, $I_{o \rightarrow s}$ and $V_{o}$ will be used to represent the short-circuit current and open-circuit voltage of the TEG module at the different operating conditions.

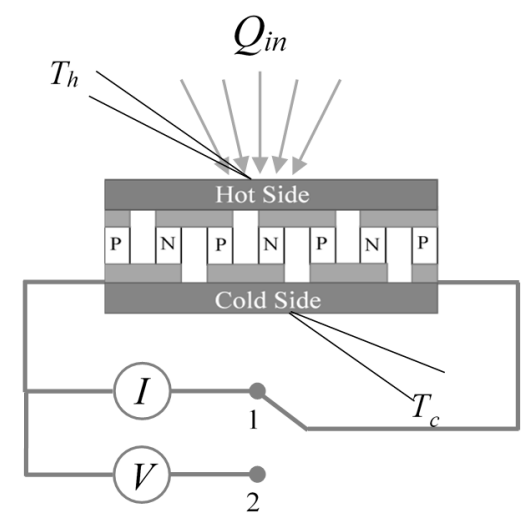

Figure 2. Diagram of the circuit used.

According to this method, the ZT is calculated by [19]:

$$
\mathrm{ZT}=\frac{I_{o \rightarrow s}}{I_{S}}-1
$$

The maximum power obtained from the TEG is calculated from Equation (7).

$$
P_{\text {max }}=\frac{1}{4} I_{S} \cdot V_{o}
$$




\section{Results and Discussion}

Measurements have been performed on the three TEGs listed in Table 1. The concentrated light was incident from a concentrated solar simulator with two different intensities of $12 \times$ and $105 \times$. The ratio of the illuminated area of the TEG, which was controlled by the galvanized steel masks, varied between $10-80 \%$.

The open-circuit voltage, $V_{o}$, has been recorded for the two mentioned illumination levels as depicted in Figure 3. The output voltage of the 4-cm TEG (sample B) showed the highest values amongst all the three TEGs under both high and low concentrated light levels. This was attributed to the higher number of P-N junctions of such a sample (127 junctions). The 6.2-cm TEG (sample C) generated a higher voltage than that of the $3-\mathrm{cm}$ one (sample A) in low concentrated light intensity, while they were nearly similar in the case of high concentrated light intensity.

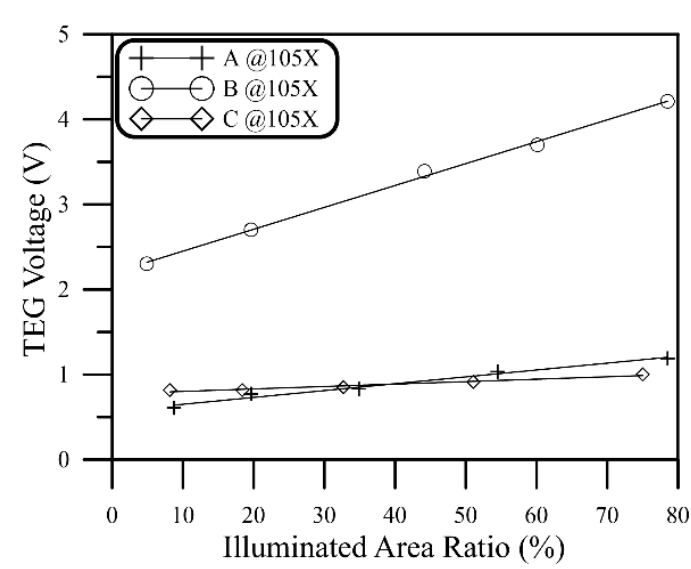

(a)

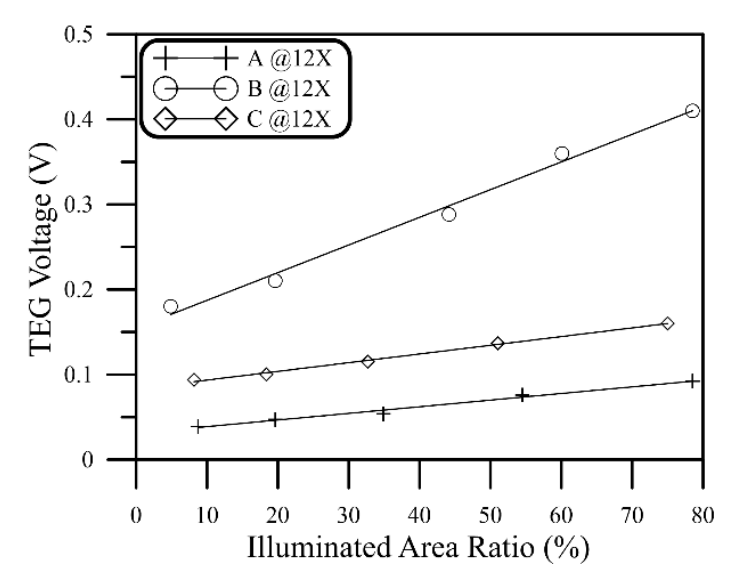

(b)

Figure 3. TEG output voltage of the three samples, A (3-cm TEG), B (4-cm TEG), and C (6.2-cm TEG), as a function of percentage of the TEG-illuminated hot-side area for (a) $105 \times$ and (b) $12 \times$.

The generated open-circuit voltage divided by the total number of junctions for each the three TEG samples in both high and low concentrated light intensities is shown in Figure 4. The generated voltage was highest for the 3-cm TEG (sample A), then for the 4-cm TEG (sample B), and finally for the 6.2-cm TEG (sample C) at high concentrated light, as illustrated in Figure 4a. However, such behavior was reversed when operating under low concentrated light, as shown in Figure $4 \mathrm{~b}$.

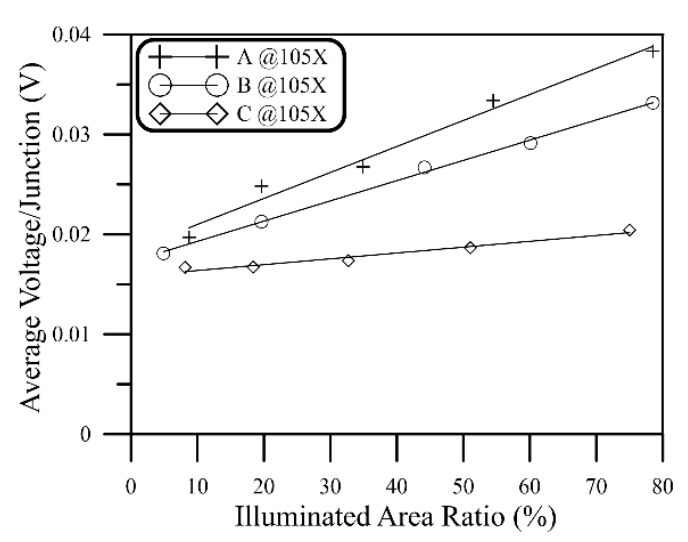

(a)

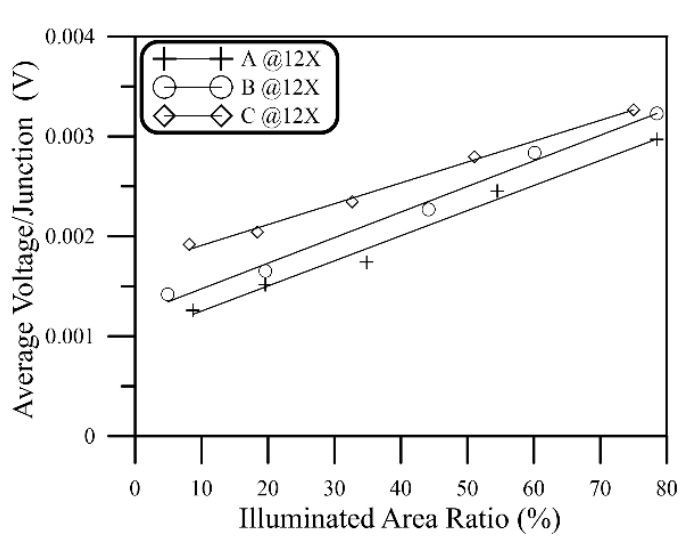

(b)

Figure 4. Increase in average voltage per number of junctions of the three samples, A (3-cm TEG), B (4-cm TEG), and C (6.2-cm TEG), with increasing the percentage of illumination covering of the TEG surfaces at (a) $105 \times$ and (b) $12 \times$. 
To better understand this behavior, the electrical performance of the TEGs on hand, the nature of the junction connections inside the TEGs, and the temperature distribution over the TEG surface have been further investigated.

Figure 5a shows the P-N junctions of a TEG module. Each P-N junction has an equivalent circuit consisting of a voltage source and junction internal resistance, as demonstrated in Figure $5 \mathrm{~b}$. All junctions are connected in series as shown in Figure 5c. In a series connection of many voltage sources, voltages are summed up to give the total voltage of the system, or the TEG open-circuit voltage. Such voltage depends on the temperature distribution over the TEG surface. It varies significantly if all the junctions of the TEG are not exposed to the same temperature difference. The Seebeck coefficient as well as internal resistance of the $\mathrm{P}$ - and $\mathrm{N}$ - thermos-elements will also vary with such uneven temperature difference applied to the junctions.

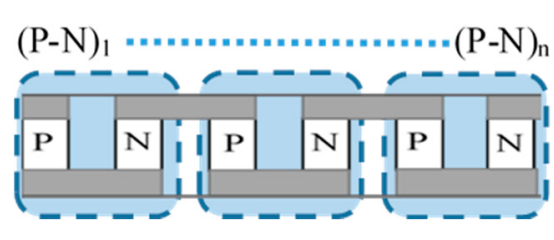

(a)

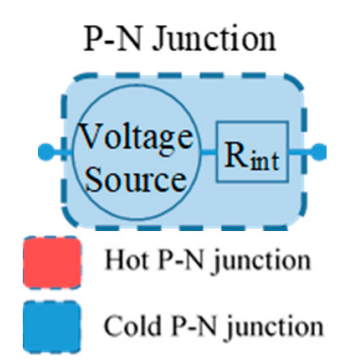

(b)

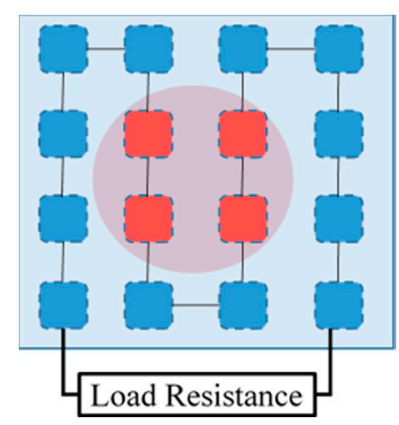

(c)

Figure 5. Schematic diagram for (a) P-N junctions of a TEG module, (b) equivalent circuit of P-N junction, and (c) centrally concentrated light on the TEG surface showing hot and cold series-connected P-N junctions.

CFD simulations for investigating the temperature distribution on PV cells or TEGs have significant impacts on interpreting their results and predicting performances. An example is the three-dimensional simulation performed on perovskite solar cells [21].

A rough CFD simulation has been carried out in this research to investigate the variation in temperature across the TEG area on the device performance, and thus explain the obtained results mentioned above. Simulations have been carried out for both the 3-cm and the 6.2-cm TEGs (sample A and sample C) operating under two light intensities of $12 \times$ and $105 \times$. Figure 6 shows a CFD simulation of the 3-cm TEG exposed to a centrally concentrated light zone with a diameter of $2 \mathrm{~cm}$ and intensity of $12 \times$. The upper ceramic was separated from the remaining TEG components in the figure for illustrative purposes only.

From the CFD simulation, temperatures were collected from two selected junctions, one hot junction in the middle of the illuminated zone, and the other is an unilluminated cold junction near the TEG edge. Temperatures of four points of concern were collected, namely:

- Point 1 , located in the middle of the illuminated zone, on the TEG's hot side;

- Point 2 (not shown in the figure), which is opposite to Point 1 and is located on the cold (bottom) side;

- Point 3, located near the hot side's edge;

- Point 4, which is opposite to Point 3 and is located on the cold side. 


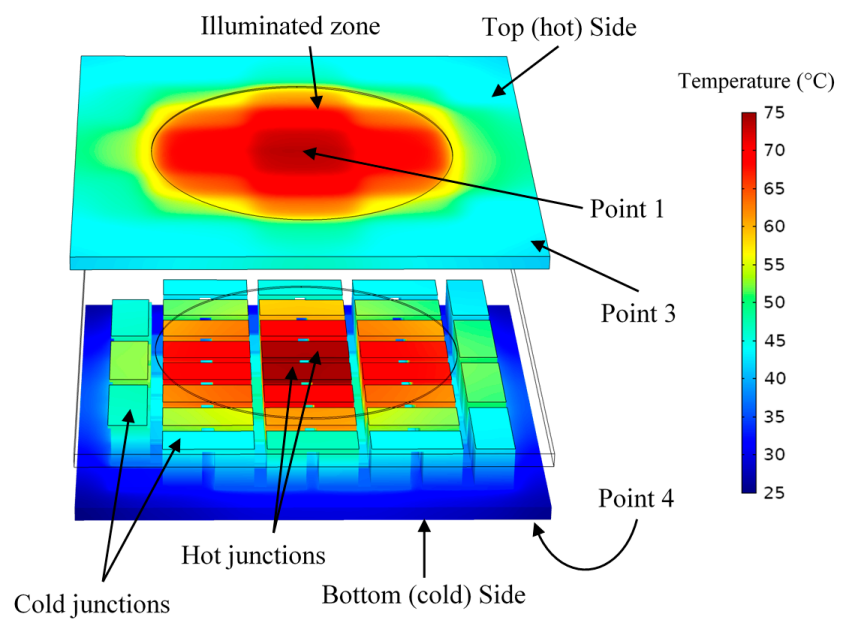

Figure 6. CFD simulation of the 3-cm TEG (sample A) operating under a concentrated light zone with a diameter of $2 \mathrm{~cm}$ and intensity of $12 \times$. Point 1 is in the middle of the TEG's illuminated zone on the hot side; Point 2 (not shown) is opposite to Point 1 and on the cold side; Point 3 is near the hot-side edge; Point 4 is opposite to Point 3 and on the cold side.

The complete simulation results and the prediction of TEG performance under such partial illumination conditions and validation of simulations against lab measurements will be published in a separate paper.

Table 2 gives the predicted values of the hot and cold junction temperatures of the $3-\mathrm{cm}$ and the 6.2-cm TEGs operating under $12 \times$ and $105 \times$ with an illuminated zone diameter of $2 \mathrm{~cm}$. This illuminated zone represents an illumination covering percentage of about $35 \%$ and $8 \%$ relative to the total hot-side area of the $3-\mathrm{cm}$ and the $6.2-\mathrm{cm}$ TEGs, respectively. At an illumination of $105 \times$, the temperature difference $\Delta T$ of both the hot (junction inside the illuminated zone) and cold junctions (junction far from the illumination) were higher in the 3-cm TEG compared to that of the $6.2-\mathrm{cm}$ one. Thus, the former is expected to produce a higher voltage/junction. In contrast, at an illumination of $12 \times, \Delta T$ values of both the hot and cold junctions were significantly smaller for both TEGs.

Table 2. Hot and cold junction temperatures of the 3-cm (sample A) and the 6.2-cm (sample C) TEGs operating under an illumination of $12 \times$ and $105 \times . \Delta \mathrm{T}$ (hot junction) and $\Delta \mathrm{T}$ (cold junction) are the temperature difference across each junction.

\begin{tabular}{|c|c|c|c|c|c|c|c|c|}
\hline \multirow{2}{*}{ TEG Sample } & \multirow{2}{*}{ Side Length $(\mathrm{cm})$} & \multirow{2}{*}{ Light Intensity } & \multicolumn{4}{|c|}{ Point Number } & \multirow{2}{*}{$\begin{array}{c}\Delta T \\
\text { (Hot Junc.) }\end{array}$} & \multirow{2}{*}{$\begin{array}{c}\Delta T \\
\text { (Cold Junc.) }\end{array}$} \\
\hline & & & 1 & 2 & 3 & 4 & & \\
\hline \multirow{2}{*}{ A } & \multirow{2}{*}{3} & $12 x$ & 70 & 49 & 41 & 36 & 21 & 5 \\
\hline & & $105 x$ & 423 & 253 & 198 & 137 & 170 & 61 \\
\hline \multirow{2}{*}{$\mathrm{C}$} & \multirow{2}{*}{6.2} & $12 x$ & 45 & 31 & 29 & 26 & 14 & 3 \\
\hline & & $105 x$ & 206 & 100 & 61 & 47 & 106 & 14 \\
\hline
\end{tabular}

This interprets the obtained results of the TEG voltage/junction output order shown in Figure 4 (the 3-cm TEG, then the 4-cm TEG, then the 6.2-cm TEG) operating under 105× illumination. In the 3-cm TEG, distances between the central (hot) zone and the (relatively colder) edges are smaller than those in the $6.2-\mathrm{cm}$ one. Hence, variations in temperature difference between the hot and cold junctions are lower compared to those in the 6.2-cm TEG, resulting in a relatively high module voltage (although with a lower number of junctions).

In contrast, the current generated from the TEG behaves differently. Figure $7 \mathrm{a}, \mathrm{b}$ show the measured short-circuit current of each of the TEGs operating under illumination intensities of 105x and $12 \times$, respectively. Referring to Figure 5, and recalling that the TEG module consists of a number of series-connected P-N junctions, the current of a TEG is the current generated based on the total resistance of those series-connected junctions. Although both the $3-\mathrm{cm}$ and the $6.2-\mathrm{cm}$ modules 
generate almost the same voltage when operating at a high light intensity-as already discussed in Figure 3a-the 3-cm module current shown in Figure 7a is higher because of its lower internal resistance ( $0.18 \Omega$ at room temperature) compared to that of the $6.2-\mathrm{cm}$ one $(0.22 \Omega$ at room temperature). This behavior is reversed when operating under low light intensity, as shown in Figure $7 \mathrm{~b}$. This is because the voltage generated by the 6.2-cm TEG (sample C) is higher compared to that of the 3-cm one (sample A), as shown in Figure 3b. Hence, the current of the former is higher, as shown in Figure $7 \mathrm{~b}$. Having the highest internal resistance amongst the three TEGs on hand (2.28 $\Omega$ ), the 4-cm TEG (sample B), produced the lowest current under both low and high light intensities.

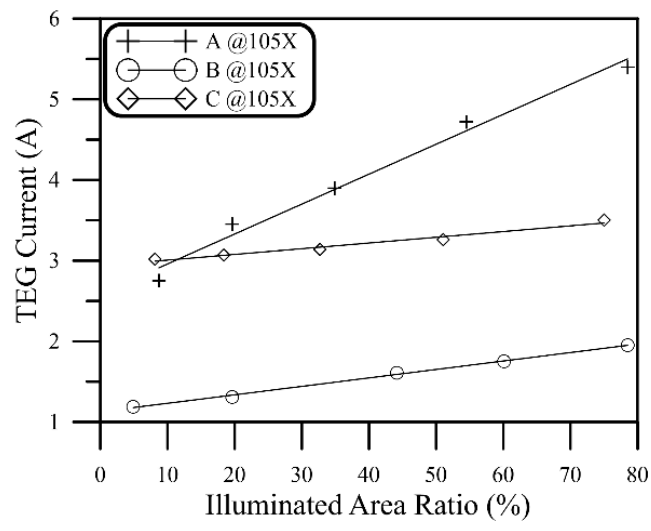

(a)

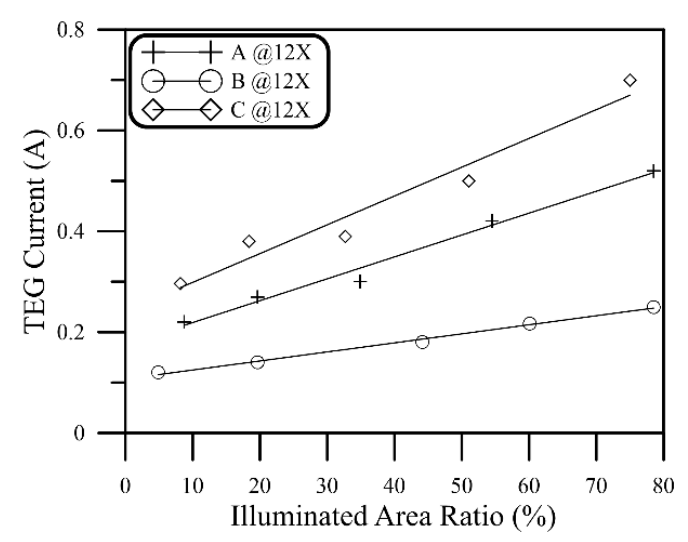

(b)

Figure 7. Short-circuit current as a function of percentage of the illuminated area on the hot surfaces of the TEG samples under light intensity of (a) $105 \times$ and (b) $12 \times$.

The figure-of-merit, ZT, has been calculated for the three TEG modules as a function of the illumination percentage of the hot-side area and the illumination intensity using Equation (6) described above. The results illustrated in Figure 8 indicates that the $Z T$ values of all modules are higher in lower light intensities (Figure 8b) compared to those under high light intensities. In both graphs, the ZT showed an asymptotic decrease while increasing the percentage of the illumination covering ratio, which results in an increase in the average TEG temperature as a whole. Referring to the definition of $Z T$ in Equation (4), the effect of increasing the illuminated area of the TEG surface (and thus increasing the average TEG temperature as a whole) on all the parameters upon which the ZT is dependent could result in a decrease in the $Z T$.

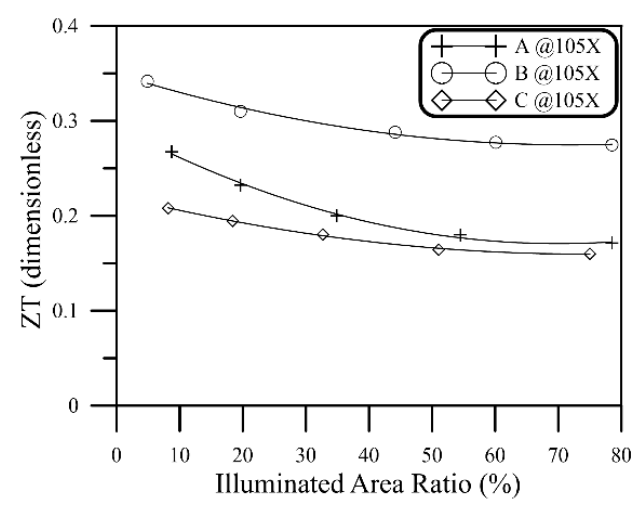

(a)

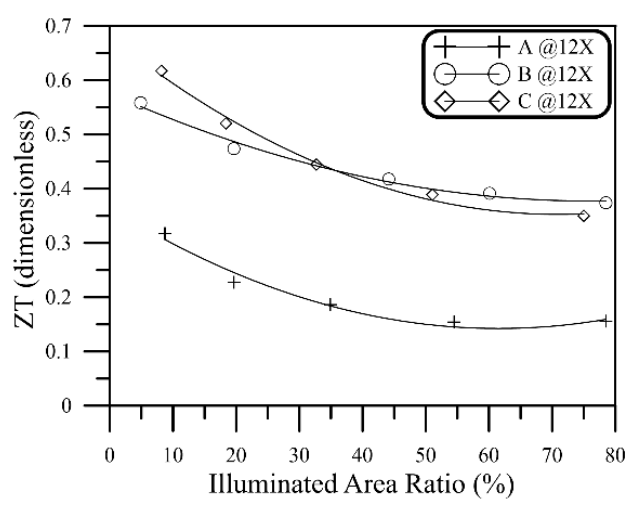

(b)

Figure 8. $\mathrm{ZT}$ as a function of the illumination area percentage for the three TEG samples under light intensity of (a) $105 \times$ and (b) $12 \times$. 
The maximum power extracted from the TEG modules under both high and low illumination intensities have been calculated according to Equation (7) and plotted as shown in Figure 9. Under 105x, the 4-cm TEG delivered the highest power amongst the three TEGs, followed by sample A. In contrast, sample C TEG delivered the highest power under light intensity of $12 \times$.

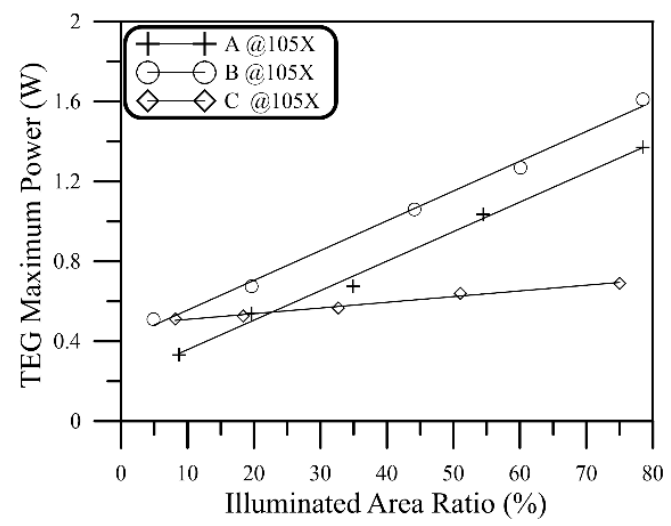

(a)

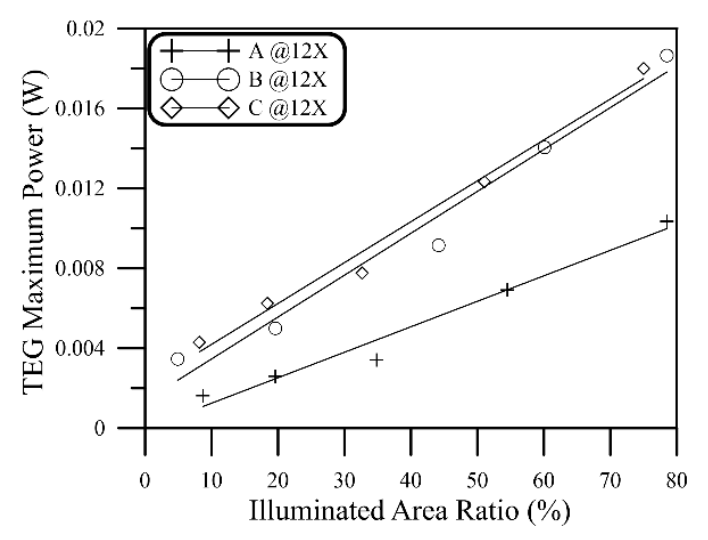

(b)

Figure 9. Maximum power of TEG samples as a function of the illuminated area percentage under light intensity of (a) $105 \times$ and (b) $12 \times$.

The impact of investigating the TEG-illuminated area ratio is signified further by the generated TEG power graphs shown in Figure 9, along with the data tabulated in Table 2. Both the 3-cm and the 6.2-cm TEGs were illuminated with 105× concentrated light, which is incident for both samples on a mask with a hole of diameter $2 \mathrm{~cm}$. The temperature at point 1 (which is assumed to be the PV temperature) reached $423^{\circ} \mathrm{C}$ in the case of the 3-cm TEG (i.e., an illuminated area percentage of $35 \%$ ), while it reached $206^{\circ} \mathrm{C}$ in the case of the 6-cm TEG (i.e., an illuminated area percentage of $8 \%$ ). This means that the PV-operating temperature is doubled if the $6.2-\mathrm{cm}$ TEG is replaced by the $3-\mathrm{cm}$ one, indicating that its efficiency will be greatly reduced (depending on the properties of the PV to be used). On the other hand, when exposed to the same light intensity, the 3-cm TEG produced 0.67 Watt, while the 6.2-cm TEG produced 0.51 Watt. This show clearly the effect of studying the TEG illumination percentage and its consequences on both TEG- and PV-produced power.

In a recent study [11], two types of CPV cells have been theoretically examined, and their electrical output has been predicted. In view of the findings of this current paper, this study will be utilized to further theoretically and experimentally study the total output of the CPV/TEG for a given CPV cell.

\section{Conclusions}

This work investigates the electrical and thermal performance of a partly illuminated TEG as a step forward towards predicting the performance of a CPV/TEG coupled system. The performance of three TEG modules with different areas was investigated by allowing concentrated solar radiation as a heating source to be incident on the hot side of the module. Two different concentrated light intensities were used: (a) 105× and (b) 12x. Each module was tested by varying the percentage of the illuminated area to the total hot-side area, while cooling the cold side by water flowing at a constant temperature. The values of the TEG voltage, current (and thus ZT) as well as maximum power behaved differently under each illumination intensity tested in this work.

To investigate this behavior, a CFD simulation was performed to study the effect of the nonuniformity of temperature distribution over the TEG module. The results of the simulation were then used to successfully explain the performance variation of these TEGs under various illuminations. Having the highest number of junctions, the 4-cm TEG (sample B) showed the highest output voltage amongst all the three TEGs under both high and low concentrated light levels. Regarding the short-circuit current output, the 3-cm TEG (sample A) operating under high light concentration was 
highest, while under low light concentration, it was the 6-cm TEG (sample C). The 4-cm TEG (sample B) gave the lowest current under both high and low light intensities due to its highest internal resistance.

Accordingly, the 4-cm TEG (sample B) delivered the maximum power under high light intensity, while this was provided by the 6-cm TEG under low light intensity when compared to the other TEG samples.

These results may provide a deeper insight towards implementing the TEG in an integrated CPV/TEG system, which serves not only to passively cool the solar cell, but also to produce extra thermoelectric power.

Author Contributions: Conceptualization, M.S.; measurements and analysis, A.L. and M.A.T. All authors have read and agreed to the published version of the manuscript.

Funding: This work was funded by National Science, Technology and Innovation Plan (MAARIFAH), King Abdulaziz City for Science and Technology (KACST), Kingdom of Saudi Arabia, grant number (14-ENE2310-10).

Conflicts of Interest: The authors declare no conflict of interest.

\section{References}

1. Geisz, J.F.; France, R.M.; Schulte, K.L.; Steiner, M.A.; Norman, A.G.; Guthrey, H.L.; Young, M.R.; Song, T.; Moriarty, T. Six-junction III-V solar cells with $47.1 \%$ conversion efficiency under 143 Suns concentration. Nat. Energy 2020, 5, 326-335. [CrossRef]

2. Mbewe, D.J.; Card, H.C.; Card, D.C. A model of silicon solar cells for concentrator photovoltaic and photovoltaic/thermal system design. Sol. Energy 1985, 35, 247-258. [CrossRef]

3. Sabry, M.; Ghitas, A.E. Influence of Temperature on Methods for Determining Silicon Solar Cell Series Resistance. J. Sol. Energy Eng. 2007, 129, 331-335. [CrossRef]

4. Kurtz, S.; Myers, D.; McMahon, W.E.; Geisz, J.; Steiner, M. A comparison of theoretical efficiencies of multi-junction concentrator solar cells. Prog. Photovolt. Res. Appl. 2008, 16, 537-546. [CrossRef]

5. Li, P.; Cai, L.; Zhai, P.; Tang, X.; Zhang, Q.; Niino, M. Design of a Concentration Solar Thermoelectric Generator. J. Electron. Mater. 2010, 39, 1522-1530. [CrossRef]

6. Djafar, Z.; Piarah, W.H. A New Hybrid of Photovoltaic-thermoelectric Generator with Hot Mirror as Spectrum Splitter. J. Phys. Sci. 2018, 29, 63-75. [CrossRef]

7. Lekbir, A.; Meddad, M.; Eddiai, A.; Benhadouga, S.; Khenfer, R. Higher-efficiency for combined photovoltaic-thermoelectric solar power generation. Int. J. Green Energy 2019, 16, 371-377. [CrossRef]

8. Li, G.; Zhang, G.; He, W.; Ji, J.; Lv, S.; Chen, X.; Chen, H. Performance analysis on a solar concentrating thermoelectric generator using the micro-channel heat pipe array. Energy Convers. Manag. 2016, 112, 191-198. [CrossRef]

9. Li, G.; Zhao, X.; Ji, J. Conceptual development of a novel photovoltaic-thermoelectric system and preliminary economic analysis. Energy Convers. Manag. 2016, 126, 935-943. [CrossRef]

10. Zhou, Y.-P.; He, Y.-L.; Qiu, Y.; Ren, Q.; Xie, T. Multi-scale investigation on the absorbed irradiance distribution of the nanostructured front surface of the concentrated PV-TE device by a MC-FDTD coupled method. Appl. Energy 2017, 207, 18-26. [CrossRef]

11. Lashin, A.; Al Turkestani, M.; Sabry, M. Concentrated Photovoltaic/Thermal Hybrid System Coupled with a Thermoelectric Generator. Energies 2019, 12, 2623. [CrossRef]

12. Andreev, V.M.; Grilikhes, V.A.; Khvostikov, V.P.; Khvostikova, O.A.; Rumyantsev, V.D.; Sadchikov, N.A.; Shvarts, M.Z. Concentrator PV modules and solar cells for TPV systems. Sol. Energy Mater. Sol. Cells 2004, 84, 3-17. [CrossRef]

13. Wu, Y.; Eames, P.; Mallick, T.; Sabry, M. Experimental characterisation of a Fresnel lens photovoltaic concentrating system. Sol. Energy 2012, 86, 430-440. [CrossRef]

14. Wang, C.-C.; Hung, C.-I.; Chen, W.-H. Design of heat sink for improving the performance of thermoelectric generator using two-stage optimization. Energy 2012, 39, 236-245. [CrossRef]

15. Muto, A.; Kraemer, D.; Hao, Q.; Ren, Z.F.; Chen, G. Thermoelectric properties and efficiency measurements under large temperature differences. Rev. Sci. Instrum. 2009, 80, 093901. [CrossRef] [PubMed]

16. Snyder, G.J.; Snyder, A.H. Figure of merit ZT of a thermoelectric device defined from materials properties. Energy Environ. Sci. 2017, 10, 2280-2283. [CrossRef] 
17. Harman, T.C. Special Techniques for Measurement of Thermoelectric Properties. J. Appl. Phys. 1958, 29, 1373-1374. [CrossRef]

18. Min, G.; Rowe, D.M. A novel principle allowing rapid and accurate measurement of a dimensionless thermoelectric figure of merit. Meas. Sci. Technol. 2001, 12, 1261-1262. [CrossRef]

19. Min, G. Principle of determining thermoelectric properties based onI-Vcurves. Meas. Sci. Technol. 2014, 25, 085009. [CrossRef]

20. McCarty, R.; Piper, R. Voltage-Current Curves to Characterize Thermoelectric Generators. J. Electron. Mater. 2015, 44, 1896-1901. [CrossRef]

21. Saxena, P.; Gorji, N.E. COMSOL Simulation of Heat Distribution in Perovskite Solar Cells: Coupled Optical-Electrical-Thermal 3-D Analysis. IEEE J. Photovolt. 2019, 9, 1693-1698. [CrossRef]

(C) 2020 by the authors. Licensee MDPI, Basel, Switzerland. This article is an open access article distributed under the terms and conditions of the Creative Commons Attribution (CC BY) license (http://creativecommons.org/licenses/by/4.0/). 